\title{
ON CLUSTER CALCULATION FOR AMORPHOUS TETRAHEDRALLY BONDED SEMICONDUCTORS
}

\author{
M. A. GRADO-CAFFARO* and M. GRADO-CAFFARO \\ Member of the Academy of Sciences of New York; Julio Palacios 11, 9-B, 28029 \\ Madrid, Spain
}

(Received 3 February 1996; In final form 29 March 1996)

This article consists of an approach to the calculation of the density of valence states in tetrahedrally bonded amorphous semiconductors and, on the other hand, the paper contains theoretical considerations on electronic spectra related to the results obtained by the authors concerning cluster calculation. The exposition is centered on III-V compounds. Moreover, operational methods are used to perform certain computations related to the previous subjects.

\section{INTRODUCTION}

For III-V compounds, it is well-known that the results of photoemission experiments involving the energy margin within a few $\mathrm{eV}$ of the band gap are related to the optical properties. In particular, the $\xi_{2}$-spectra of amorphous InSb cannot be obtained by broadening that of the crystal [1]. Other III-V compounds such as GaAs, GaP and GaSb constitute well known examples within the above context.

On the other hand, we can claim that photoemission experiments have provided much information on the electronic spectra of amorphous tetrahedrally bonded semiconductors [2], [3], [4]. In this context, the density of valence states plays an important role. This density can be obtained experimentally with monochromatized X-rays.

\footnotetext{
${ }^{*}$ Corresponding author.
} 


\section{CAlCulations}

By using extended Hückel theory, the local electronic density of states in terms of the local Green's function is given by the following expression [5]:

$$
g(\bar{r}, \mathrm{E})=-\frac{1}{\pi} \mathrm{I} m \mathrm{G}(\bar{r}, \mathrm{E})
$$

where E denotes energy and $\bar{r}$ is the position vector. From (1) and other considerations it is deduced:

$$
g(\bar{r}, \mathrm{E})=\sum_{n}\left|\psi_{n}(\bar{r})\right|^{2} \delta\left(\mathrm{E}-\mathrm{E}_{n}\right)
$$

where the eigenfunctions $\psi_{n}$ are associated with the energy eigenvalues $\mathrm{E}_{n}$, and $\delta$ denotes Dirac function.

It is well-known that the materials with spatial disorder require a special treatment. In order to calculate the density of states in such materials, the "cluster calculation" is feasible. The electronic properties of a bulk material are basically related to the characteristics of the corresponding atoms and their local environment within a distance that is of the order of the mean free path or the thermal wavelength. In the following, we will assume that the clusters considered are isolated in vacuum.

The density of states of a cluster is given by:

$$
\tilde{g}(\mathrm{E})=\iiint_{\mathrm{D}} g(x, y, z, \mathrm{E}) d x d y d z
$$

referring to cartesian coordinates. $\mathrm{D}$ denotes the domain corresponding to the cluster. Now, we establish the following approximation by means of a Taylor's expansion:

$$
g(\bar{r}, \mathrm{E}) \cong g(0, \mathrm{E})+\bar{r} \cdot\left[\nabla_{\bar{r}} g\right]_{0}
$$

where $\nabla_{\bar{r}} g$ refers to the spatial coordinates, that is: 


$$
\nabla_{\bar{r}} \equiv\left(\frac{\partial}{\partial x}, \frac{\partial}{\partial y}, \frac{\partial}{\partial z}\right)
$$

By substituting (4) into (3) we obtain:

$$
\tilde{g}(\mathrm{E}) \cong \Omega g(0, \mathrm{E})+\iint_{\mathrm{D}} \int \bar{r} \cdot\left[\nabla_{\bar{r}} g\right]_{0} d x d y d z
$$

where $\Omega$ is the volume of the cluster. Introducing the wave-functions $\psi_{n}$, from (2) we find:

$$
\begin{gathered}
g(0, \mathrm{E})=\sum_{n}\left|\psi_{n}(0)\right|^{2} \delta\left(\mathrm{E}-\mathrm{E}_{n}\right) \\
\nabla_{\bar{r}} g=2 \sum_{n}\left\{\left[\operatorname{Re} \psi_{n}\right] \nabla\left[\operatorname{Re} \psi_{n}\right]+\left[\operatorname{Im} \psi_{n}\right] \nabla\left[\operatorname{Im} \psi_{n}\right]\right\} \times \delta\left(\mathrm{E}-\mathrm{E}_{n}\right) .
\end{gathered}
$$

Remark: In the previous exposition, the point $0=(0,0,0)$ is the origin of the cluster.

By substituting (6) and (7) into (5), we obtain:

$$
\begin{gathered}
\tilde{g}(\mathrm{E})=\sum_{n}\left\{\Omega\left|\psi_{n}(0)\right|^{2}+2 \iint_{\mathrm{D}} \int \bar{r} \cdot\left[\operatorname{R} e\left(\psi_{n}\right)_{0}\left(\nabla \operatorname{Re} e\left(\psi_{n}\right)\right)_{0}+\right.\right. \\
\left.\left.\operatorname{I} m\left(\psi_{n}\right)_{0}\left(\nabla \operatorname{I} m\left(\psi_{n}\right)\right)_{0}\right] d x d y d z\right\} \delta\left(\mathrm{E}-\mathrm{E}_{n}\right)
\end{gathered}
$$

Now, it is interesting to determine the $\xi_{2}$-spectrum [6]:

$$
\xi_{2}(\omega) \cong \frac{\mathrm{C}}{\omega^{2}} \int_{0}^{\hbar \omega} \tilde{g}(\mathrm{E}) \tilde{g}(\hbar \omega-\mathrm{E}) d \mathrm{E}
$$

where $\mathrm{C}$ is a constant $\left(\hbar \omega>2 \mathrm{E}_{\mathrm{c}} ;(\mathrm{c}) \rightarrow\right.$ conduction band).

From the expression (8), it is trivial to calculate $\tilde{\mathrm{g}}(\hbar \omega-\mathrm{E})$. However, the calculation of $\xi_{2}$ is tedious if we substitute (8) directly into (9); in contrast, if we apply an operational method (e.g., the Laplace transform), the problem in question is solved.

Expression (9) can be expressed as a convolution, namely: 


$$
\xi_{2}(\omega) \cong \frac{\mathrm{C}}{\omega^{2}}[\tilde{g}(\hbar \omega)] *[\tilde{g}(\hbar \omega)]
$$

and applying Borel's convolution theorem for the Laplace's transform, we can write:

$$
\frac{1}{\hbar^{2}} \succeq\left[(\hbar \omega)^{2} \xi_{2}(\hbar \omega)\right]=\mathrm{C}\{\succeq[\tilde{g}(\hbar \omega)]\}^{2}
$$

On the other hand, it is easy to calculate the left-hand side of (11). The result is:

$$
\hbar^{-2} \longleftarrow\left[(\hbar \omega)^{2} \xi_{2}(\hbar \omega)\right]=\frac{d^{2}}{d s^{2}}\left\{€\left[\xi_{2}(\hbar \omega)\right]\right\}
$$

From (11) and (12) it is deduced:

$$
\tilde{g}(\hbar \omega)=\mathrm{C}^{\prime} \mathrm{七}^{-1}\left\{\frac{d^{2}}{d s^{2}}\left[\mathrm{E}\left(\xi_{2}(\hbar \omega)\right)\right]\right\}^{1 / 2}
$$

with $\mathrm{C}^{\prime} \equiv \mathrm{C}^{-1 / 2}$

Expression (13) permits to calculate the density of states when the $\xi_{2}$-spectrum is known from experiments.

\section{CONCLUDING REMARKS}

The approach realized by us in order to compute the density of states of a cluster is in good agreement with the experimental works related to amorphous tetrahedrally bonded semiconductors. In particular, this agreement occurs in amorphous III-V compounds [4]. The theory exposed in this paper is suitable for the study of several devices based on amorphous semiconductors. In particular, we refer to thin-film devices [7]. Moreover, the theoretical procedure established to calculate the density of states from spectrum constitutes an example of the Laplace's transform to solve problems related to spectral analysis. This procedure is consistent with references [8] [9] [10]. 
On the other hand, by changing $\hbar \omega$ by $\mathrm{E}$ in (13), it is feasible to equate (8) and (13), and from this equality, to derive various conclusions.

\section{References}

[1] C. Ribbing, D.T. Pierce, and W.E. Spicer (1972) Phys. Rev. B 4, 4417.

[2] D. E. Eastman and J. Freeouf. (1973) Solid State Commun. 13, 1815 .

[3] W. D. Grobman and D. E. Eastman. (1972) Phys. Rev. Lett. 29, 1508.

[4] N. J. Shevchik. (1974) AIP Conf. Proc. 20, 72-80

[5] B. Y. Tong. (1974) AIP Conf. Proc. 20, 148

[6] N. K. Hindley. (1970) J. Non-cryst. Solids 5, 17 .

[7] Amorphous semiconductor technologies and devices (Ed. Y. Hamakawa, NorthHolland, 1986).

[8] M. A. Grado-Caffaro and M. Grado-Caffaro. (1993) Act. Pass. Electronic Comp. 16, $49-53$

[9] M. A. Grado-Caffaro and M. Grado-Caffaro. (1993) Mod. Phys. Lett. B 7, 1201-1207

[10] M. A. Grado-Caffaro and M. Grado-Caffaro. (1992) Phys. Lett. A 169, 399-400). 

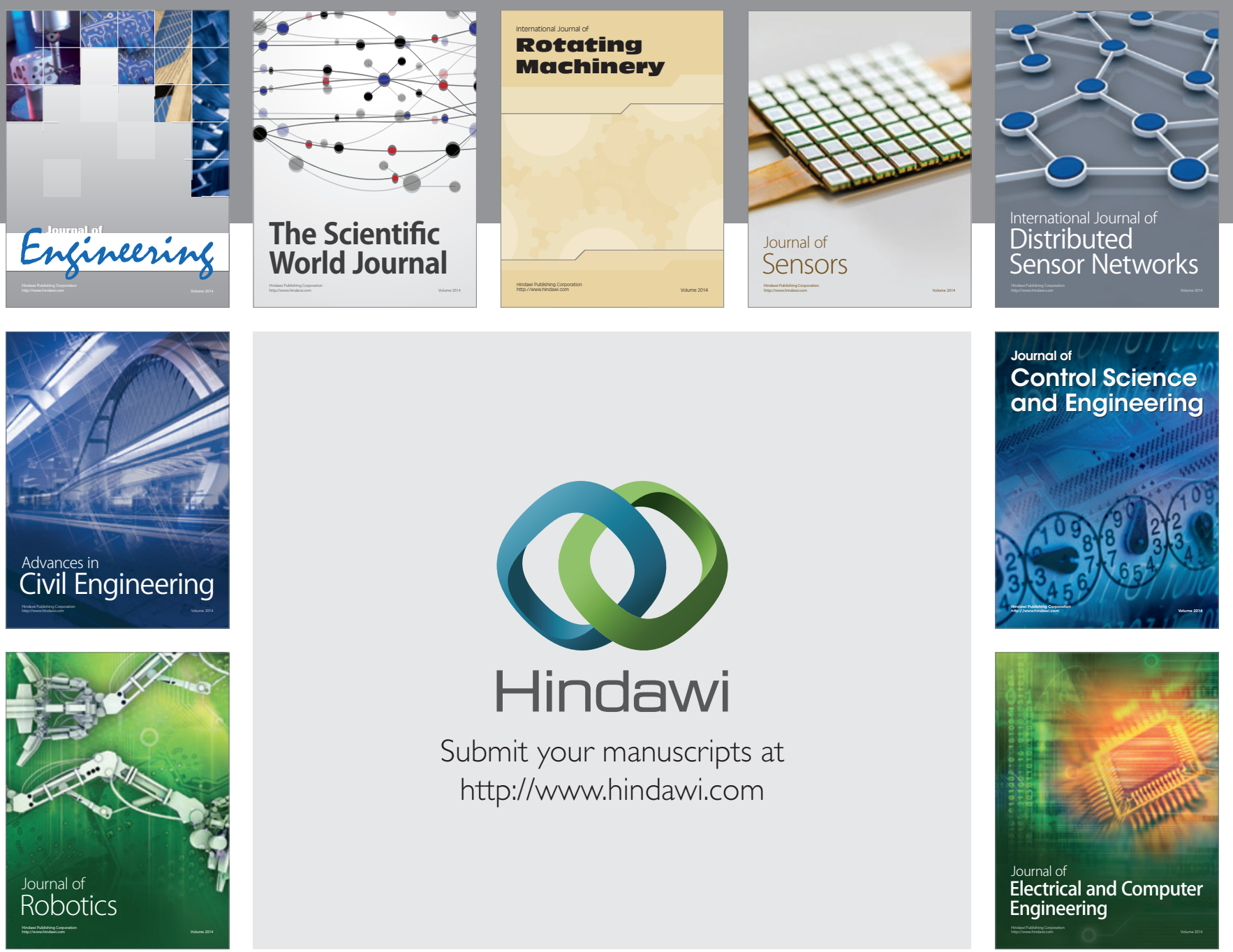

Submit your manuscripts at

http://www.hindawi.com
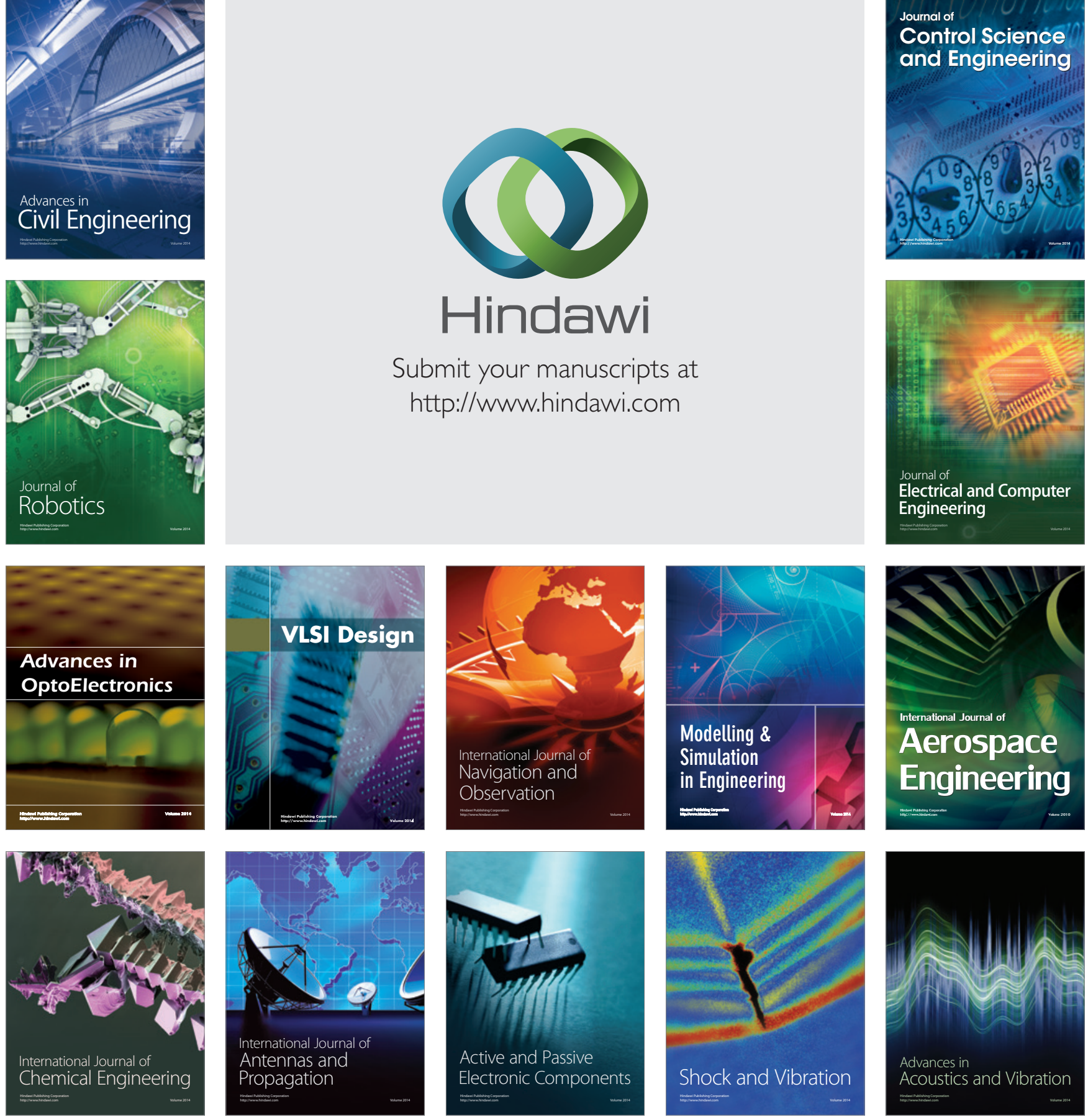